\title{
The Design for Heat Flux Sensor of High Transient and Large Range in the Explosion Field
}

\author{
Guo Yu-yan, KongDe-ren* and Chen Jing \\ School of Mechanical Engineering \\ Nanjing University of Science and Technology \\ Guo_Yu_yan@163.com,derenkongnj@sina.com,c0819j@126.com
}

\begin{abstract}
In this paper, the characteristics of the thermal damage in explosive field have been analyzed, and the technical indicators of the heat flux sensors which are used for thermal damage test have been pointed out. The structure of Gardon gauge was modeled and analyzed. And the mathematical model of Gardon gauge was improved on this basis. By expanding the mathematical model to the non-steady heat transfer stage, the response speed of the Gardon gauge is increased immensely. And also the structure of Gardon gauge was improved to meet the need of the thermal damage test in the explosion field. At last, a numerical simulation was performed on the improved gauge, which verified the feasibility of applying this gauge to the thermal damage test in the explosion field.
\end{abstract}

Keywords: fast response speed, large range, Gardon gauge, heat flux sensor

\section{Introduction}

In recent years, the thermobaric warhead gets a substantial development with the progress of technology of ammunition. Thermobaric warhead is one of warheads which mainly use the temperature and pressure effect to produce killing effects. After the warhead exploded, there will be an intense combustion, and a lot of heat will be spread around to damage the target. Therefore the thermal damage is an important damage way of the warhead.

The thermal damage of the thermobaric warhead can generally be divided into three categories [1]: (1) Thermal radiation to the target caused by blast fireball. (2) The rise of temperature caused by an adiabatic compression of the gas from shock wave. (3) The heat conduction and thermal convection caused by the high temperature products after the explosion.

As a result of the differences among the mechanism of three damage ways, the characteristics and ranges of these damages are also different.

In the first way, the thermobaric warhead damages the target by the way of thermal radiation. The radiation heat transfer does not require direct contact with the target. Therefore the damage caused by it is quick and wide. But this damage has a shorter duration because the blast fireball is fleeting. In the second and the third way, the thermobaric warhead makes the damage to the target in the form of thermal convection. So it requires the direct contact with the target to make thermal damage. So in these two ways, the damage has longer effect time on closer target.

As one of the important damage effects of thermobaric warhead, the thermal damage gets more and more attention. And the test technology of thermal damage has been rapid development in recent years. At present, it mainly uses the heat flux to value the degree of thermal damage in explosion field. The testing method of heat flux in explosion field can

* Corresponding Author 
be divided into contact testing method and the non-contact testing method. The non-contact testing method uses the optical instruments, such as the infrared thermometer, to test the thermal effects. It can only analyze the heat transfer of the fireball surface, and can not get the convective heat transfer of the explosion field. So it can not be evaluation of thermal damage effects comprehensively. In addition, it will face the problem that the emissivity of explosion products is unknown and the measuring instruments are easily interfered by dust and smoke. In view of the above reasons, researchers are beginning to use the contact testing method in recent years.

The heat sensitive component in the contact testing method is thermocouple, thermal resistance and so on. The contact testing method makes the test to the heat flux of explosion field by the way of contacting with explosion products. The duration of thermobaric explosive field only last several hundred milliseconds. And the release of heat flux can reach megawatts per square meter [2,3]. So it requires heat flux sensors have a small response time and large measuring range. And that is the bottleneck of the contact testing method at present.

The differential sensor is one of the popular types of heat flux sensor. By measuring the temperature drop across a known non-metal thermal resistance, the heat flux through the sensor is determined. This sensor is used Fourier's law, which for 1D steady state, to measure heat flux. Because of the small thermal conductivity and weak high-temperature tolerant of the non-metal thermal resistance, the response speed of the differential sensor is slow and the range is not wide [4,5]. Thus it affects its uses in the high-temperature explosion field.

Gardon gauge is another kind of heat flux sensor used Fourier's law. The heat flux which received by the Gardon gauge can be calculated according to the temperature differences between the circular foil center and the surrounding heat sink. Different from the differential sensor, the thermal resistance layer of Gardon gauge is metal. In addition, the response speed of Gardon gauge is improved with the wider range. Therefore Gardon gauge have congenital advantages in the thermal damage tests.

Therefore this paper improve the traditional Gardon gauge according to the characteristics of the explosion field, so that it can reach the range of megawatts per square meter and also has a quickly response. Finally, the rationality of the sensor will be verified by numerical analysis.

\section{The Improvements of Gardon Gauge}

\subsection{The Test Principles of Gardon Gauge}

Gardon gauge is also called circular foil heat flux transducer, and its structure diagram is shown in Figure 1. The circular constantan foil and copper heat sink is fixed together by welding, and each one is connected by a copper wire. So each the center (the contact point of constantan and copper wire) and the edge (the contact point of constantan and heat sink) of the constantan foil forms a thermocouple. And two thermocouples in series form a thermopile. 


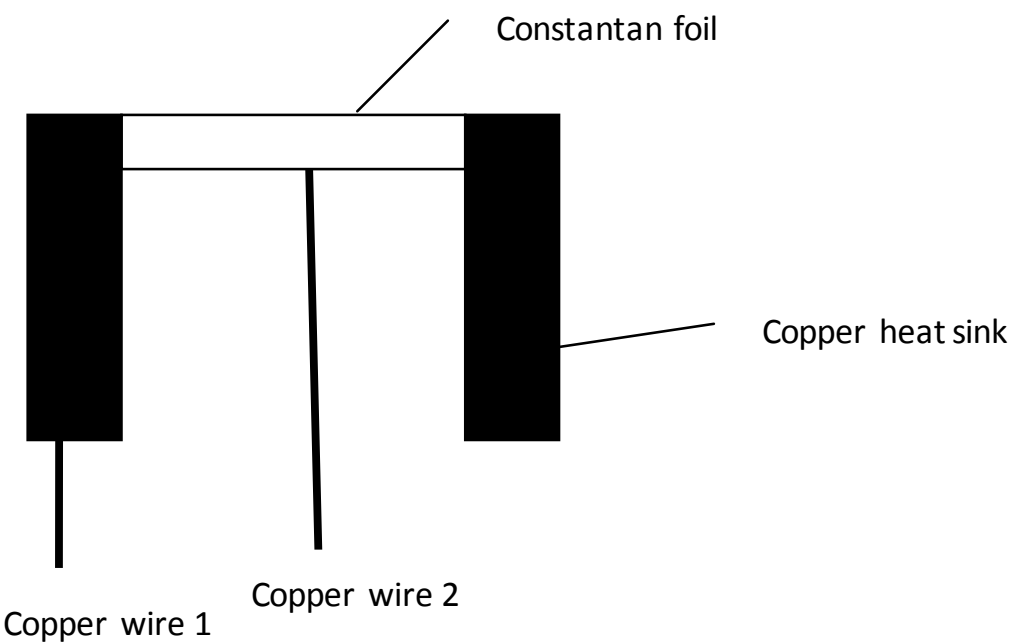

Figure 1. The Structure of Gardon Gauge

While testing, the constantan accepts heat flux from outside and transfers them to the heat sink. Initially, the heat absorption rate of constantan is greater than the heat dissipation rate, so constantan's temperature increases with the time in the initial stage, and this stage is called non-steady state. With the passage of time, the rate of heat dissipation increases gradually. When the heat absorption rate is equal to the dissipation rate, the diss ipated and absorbed energy of the constantan reach the dynamic equilibrium, and the constantan's temperature does not change with time. This stage is called steady state. Due to the heat conducts along the constantan radial, the center temperature of constantan will be higher than the edge temperature when the constantan reaches the steady state. The temperature difference between the center and edge will be calculated through the output voltage of thermopile. And then the incident heat flux can be calculated.

Because the constantan is very thin, the axial heat conduction can be ignored. And it can also assume that the temperature of constantan edge does not change with time. In the function of constant heat flux $\mathrm{q}$, when the constantan reaches to steady state $(\partial T(r, t) / \partial t=0)$, the radial temperature distribution can be represented as followed [6]:

$T(r)=\frac{q}{4 k L}\left(R^{2}-r^{2}\right)+T_{*}$

Where $T(r)$ is the temperature where the radius of constantan is $\mathrm{r} ; \mathrm{q}$ is the incident heat flux; $\mathrm{k}$ is the thermal conductivity of constantan; $\mathrm{R}$ is the external diameter of constantan; $\mathrm{L}$ is the thickness; $T_{*}$ is the edge temperature of constantan.

The expression of incident heat flux can be represented as followed by sorting function (1):

$q=\frac{4 k L \Delta T}{R^{2}}$

Where $\Delta T$ is the temperature difference between the center and edge of constantan. 
Finally Gardon gauge changes the temperature difference to electric potential difference and the incident heat flux q can be represented as followed:

$$
q=C E \ldots \ldots
$$

Where $\mathrm{C}$ is the modified constant; $\mathrm{E}$ is export potential of Gardon gauge.

According to the above knowledge, the mathematical model of Gardon gauge can only apply to the steady state. But the constantan need take some time to reach the thermal steady state in the actual measurement. So when the constantan is at non-steady state, the Gardon gauge can not accurately measure the incident heat flux. As shown in Figure 2. Therefore, the time of non-steady state (the response time of Gardon gauge) limits the varying frequencies of incident heat flux immediately.

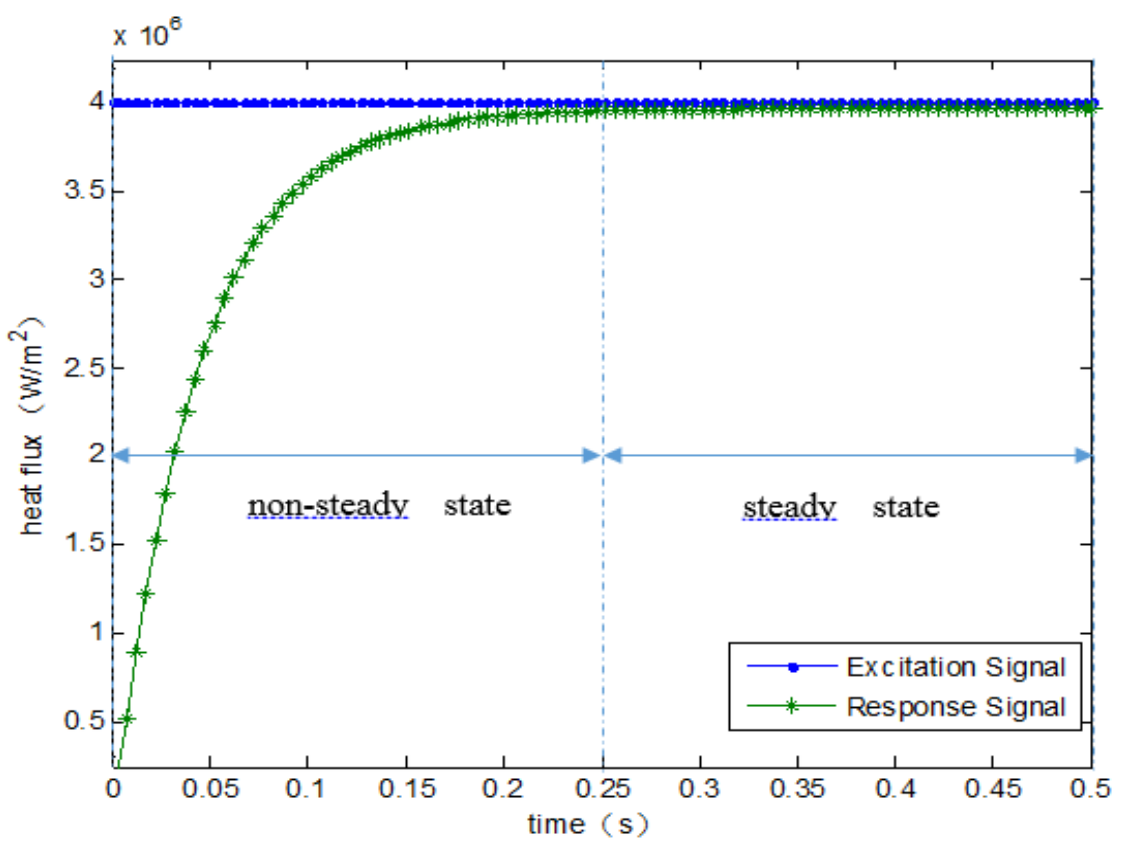

Figure 2. The Test Curve of Gardon Gauge

The duration of explosion field is short and the varying frequency of heat flux is fast. And the shorter the response time, the higher the testing precision is. The response time of the Gardon gauge is related to its diameter. And the smaller the diameter of the Gardon gauge, the faster the response rate is. At present, the smallest Gardon gauge is made by the Vatell Company which is only 1.524 millimeters in diameter, and the response time of this Gardon gauge is about 0.2 seconds [7]. So the response time is large for thermal damage test in the explosion field. In order to meet the need of thermal damage test, the response speed of the Gardon gauge is need to be improved.

There are two methods to improve the response speed of sensors. The one is decreasing the time of non-steady state. The other is extending the application range of mathematical model from the steady state to non-steady state. The former need decrease the radius of constantan. But if the radius is too small, the sensitivity of sensors will be too low. And the manufacturing difficult of sensors will be increased. In order to improve the response speed under the premise of not affecting the sensitivity of sensors, this paper uses the second method to increase the response speed. 


\subsection{The Improvements of Mathematical Model}

Non-steady state is the state in which the internal temperature of constantan varies with the time $(\partial T(r, t) / \partial t \neq 0)$. So the $\partial T(r, t) / \partial t \neq 0$ can be seen as a symbol of the non-steady state. In order to extend the application range of mathematical model from steady state to non-steady state, $\partial T(r, t) / \partial t \neq 0$ should be put in [8,9].

In the measurement, constantan received heat from its top surface, one part of the heat converted to the internal energy of the constantan, and the other heat is passed along the radial direction and eventually flows into the heat sink in the constantan edge. So this state can be expressed as follows:

$2 \pi R L q_{1}(t)=\pi R^{2} q-\pi R^{2} q_{s}(t)$

Where $\mathrm{R}$ and $\mathrm{L}$ are the radius and thickness of constantan; $\overline{\mathrm{q}_{1}}$ is the heat flux dissipated by the heat sink; $q_{s}(t)$ is the heat flux which converted to the constantan's internal energy; $\mathrm{q}$ is the heat flux received by the constantan from the environment;

The $q_{s}(t)$ can be expressed as follows:

$q_{s}(t)=\rho c L \frac{d T}{d t}$

Where $\mathrm{T}$ is the temperature of constantan at the time $\mathrm{t} ; \rho$ and $\mathrm{c}$ is the density and the specific heat of constantan.

So Equation (4) can be transformed to the form:

$2 \pi R L \overline{q_{1}}(t)=\pi R^{2} q-\pi R^{2} \rho c L \frac{d T}{d t}$

Then study the part of constantan, as Figure 3 shows.

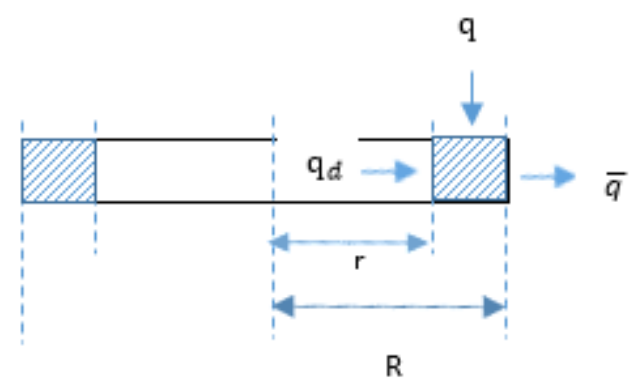

Figure 3. The Circular Ring

This part can be seen as a circular ring whose inner radius is $r$ and outer radius is $R$. When the constantan reaches the thermal steady state, the dissipated and absorbed energy of the ring reach the dynamic equilibrium, this state can be expressed as follows:

$$
2 \pi R L \overline{q_{2}}=\pi\left(R^{2}-r^{2}\right) q+2 \pi r L q_{d}(r) \ldots \ldots
$$

Where $\mathrm{r}$ and $\mathrm{R}$ are the inner diameter and outer diameter of the circular ring; $\mathrm{L}$ is the thickness; $\overline{q_{2}}$ is the heat flux dissipated by the heat sink; $\mathrm{q}$ is the heat flux into the 
constantan from the top surface; $q_{d}(r)$ is the heat flux which flows into the circular ring from the inner surface.

By Equation (2), the $q_{d}(r)$ can be expressed as follows:

$q_{d}(r)=-k \frac{d T(r)}{d r}=\frac{2 k r \Delta T}{R^{2}}$

Where $\mathrm{k}$ is the thermal conductivity and $\Delta T$ is the temperature difference between the center and the edge of constantan.

So Equation (7) can be transformed to the form:

$2 \pi R L \overline{q_{2}}=\pi R^{2} q+\left(\frac{4 k L \Delta T}{R^{2}}-q\right) \pi r^{2}$

When the constantan is at steady state.

$q=\frac{4 k L \Delta T}{R^{2}}$

At this state, $\overline{\mathrm{q}_{2}}$ is a constant value, and Eq.(9) can be expressed as follows :

$2 \pi R L \overline{q_{2}}=\pi R^{2} q$

When the constantant is at non-steady state.

$q \neq \frac{4 k L \Delta T}{R^{2}}$

So at this state, $\overline{q_{2}}$ is a parameter which related to $r$ and t. and Eq.(9) can be expressed as follows:

$2 \pi R L \overline{q_{2}}(r, t)=\pi R^{2} q+\left[\frac{4 k L \Delta T(t)}{R^{2}}-q\right] \pi r^{2}$

Both $\overline{q_{1}}$ and $\overline{q_{2}}$ represent the heat flux which flows into the heat sink, and the optimum calculating result shows that they are most similar when $\mathrm{r}=3 / 4 \mathrm{R}$ with knowing the

$\rho, c 、 k 、 L 、 R$

. As Figure 4 shows. 


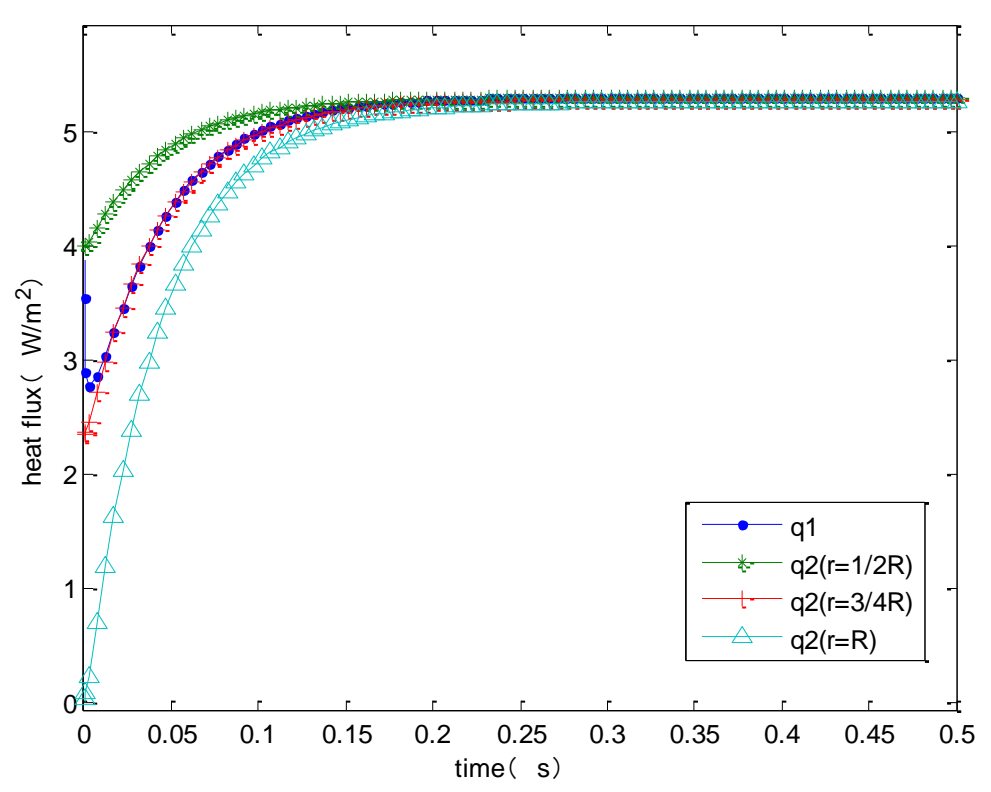

Figure 4 . The Optimum Calculating Result

In order to put in $d T / d t$, it can be assumed that $\overline{q_{1}}(3 / 4 R, t)=\overline{q_{2}}(t)$, and the incident heat flux can be expressed as follows:

$q=\frac{4 k L \Delta T}{R^{2}}+\frac{16}{9} \rho c L \frac{d T}{d t}$

Eqation (14) is the improved mathematical model. By putting in $d T / d t$, the application range of this model is extended from thermal steady state to unsteady state. And this equation shows that the incident heat flux is equal to the sum of heat flux absorbed by the constantan and heat flux pasted the constantan. The accuracy of the method will be verified by numerical simulations in the third part.

\subsection{Structure Improvement}

The traditional Gardon gauge can only test the temperature difference between the center and the edge of constantan. It can't test the temperature of constantan, and then can't get the ${ }^{d T / d t}$. So in order to fit Equation (14), the traditional Gardon gauge should be improved.

As Figure 5 shows, a constantan wire (wire 3) is connected at the center of Gardon gauge. While texting, the wire 2 and wire 3 form a thermocouple, and it can be used to measure the constantan temperature. And the wire 1 and wire 2 can be used to get the temperature difference between the center and the edge of constantan. 


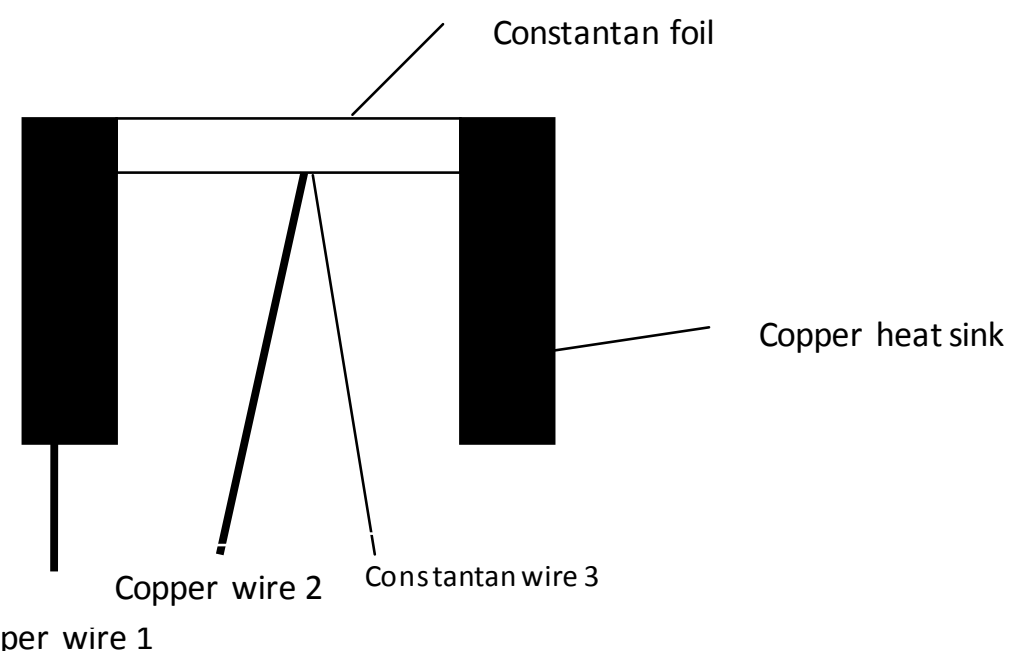

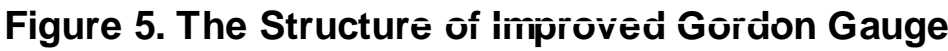

\section{Simulation Anal ysis}

In order to meet the explosive field heat flux testing demand, a Gardon gauge was designed as shown in Figure (5). The diameter and thickness of the constantan foil are $1.5 \mathrm{~mm}$ and $0.1 \mathrm{~mm}$ respectively. And the response time, maximum range and thickness of the Gardon gauge was simulated to validate its feasibility.

\subsection{The Response Time Verification}

From the above, it can be known that the duration of the explosion field is hundreds of milliseconds. In order to meet the needs of the test, the response time of the sensor should be around 0.01s. So a numerical simulation was made to show the response time of the Gardon gauge. As shown in Figure 6:

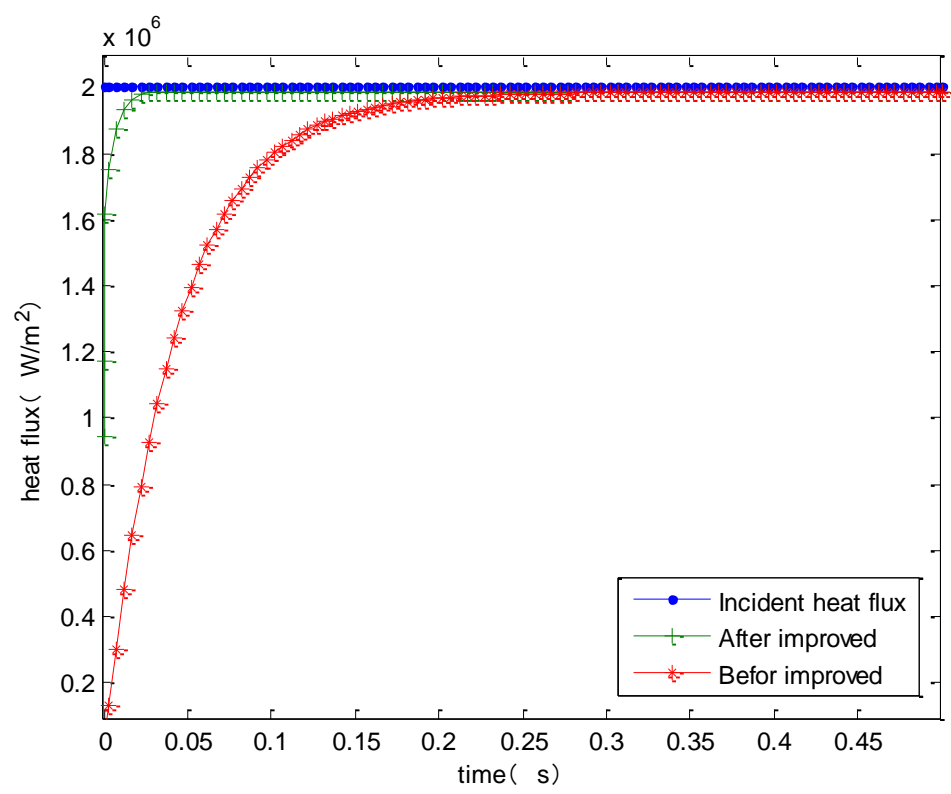

Figure 6. The Response Time of the Gardon Gauge

From the Figure 6 it can be seen that the response time of the improved Gardon gauge 
is significantly reduced, which from $0.25 \mathrm{~s}$ to $0.017 \mathrm{~s}$, when the sensor receives a step signal. That suggests the new mathematical model can improve the response speed of the sensor greatly, and that the response time of the improved Gardon gauge meets the needs of the explosive field test.

\subsection{The Measuring Range Verification}

Normally, the voltage output of the Gardon gauge should be less than $10 \mathrm{mV}$ [10]. This means that the temperature difference between the center and the edge of constantan should be less than $190^{\circ} \mathrm{C}$. When the temperature difference exceeds $285^{\circ} \mathrm{C}$, the usage of Gardon gauge should be reduced, and when it exceeds $380^{\circ} \mathrm{C}$, the Gardon gauge should be re-calibration in the next time.

So a numerical simulation was made to show the maximum temperature difference between the center and the edge of constantan under different heat flux. As shown in Table 1:

Table 1. The Maximum Temperature Difference

\begin{tabular}{|c|c|c|c|}
\hline Heat flux $\left(M W / m^{2}\right)$ & 1 & 1.5 & 2 \\
\hline $\begin{array}{c}\text { The maximum } \\
\text { temperature difference }\left({ }^{\circ} \mathrm{C}\right)\end{array}$ & 126.8 & 190.2 & 253.6 \\
\hline
\end{tabular}

It can be seen in the table (1) that the maximum temperature difference in constantan under the $2 \mathrm{MW} / \mathrm{m}^{\wedge} 2$ heat flux is only $253.6^{\circ} \mathrm{C}$, less than $285^{\circ} \mathrm{C}$. So the measuring range of improved Gardon gauge is up to $2 \mathrm{MW} / \mathrm{m}^{\wedge} 2$, and it can meet the needs of the explosive field heat damage test.

\subsection{The Thickness Verification}

Excessive thickness will lead to serious axial heat transfer, and this will affect the accuracy of test. So a numerical simulation is made to show the maximum temperature difference between the inner and outer surfaces of constantan under the $2 \mathrm{MW} / \mathrm{m}^{\wedge} 2$ heat flux. As Figure 7 shown, the difference between the two is less than $2 \%$, so the effect of the axial heat transfer can be neglected.

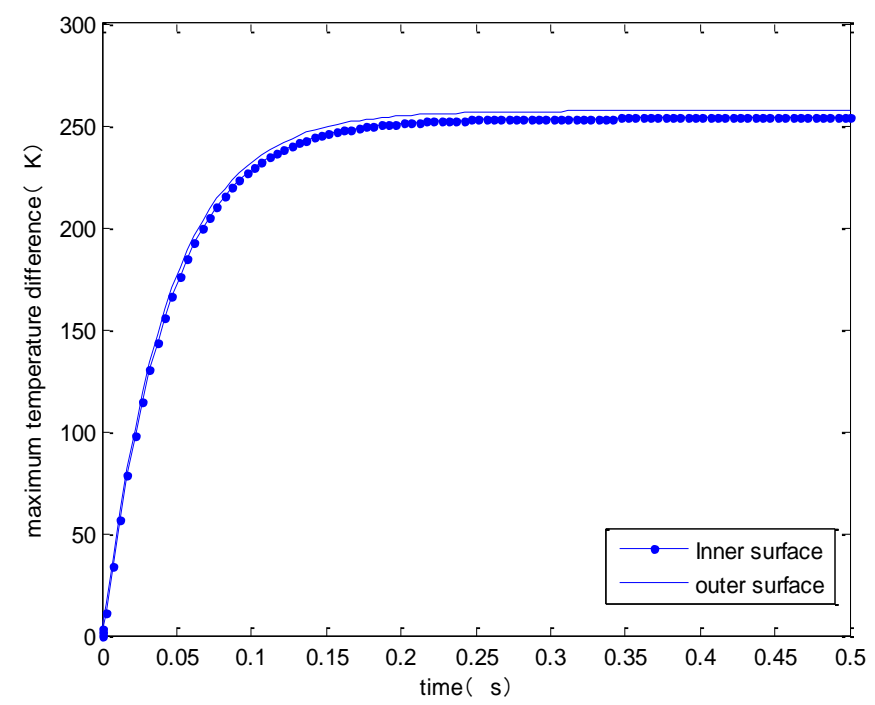

Figure 7. The Temperature Difference between the Inner and Outer Surfaces 


\section{Conclusion}

According to the above analysis, the following conclusions can be concluded:

1) The rationality of the improved Gordon's mathematical model is verified by numerical analysis. The response time of the improved Gardon gauge is reduced to about one order of magnitude.

2) The improved Gardon gauge can be used in the thermal damage test of explosive field.

3) The results of this paper are just theoretical and numerical analysis results, the actual performance of the sensor need further experimental verification.

\section{References}

[1] H. Lei, H. Zhong-qi, L. Chun-guan, Z. You-pin and C. Wang-hua, "Application of Heat Flux Microsensor in Radiation Measurement of Blasting Field", Chinese J. Explosives \& Propellants, (2011).

[2] P. Dearden, New Blast Weapons, (2015), p. 4123-37.

[3] G. Xue-yong, LI Bin and XIE Li-feng, Study on Thermal Damage of Thermobaric Explosive, Chinese Journal of Explosives \& Propellants, (2008);31: pp. 16-9.

[4] J. Ewing , A. Gifford, D. Hubble, P. Vlachos P, A. Wicks and T. Diller," A direct-measurement thin-film heat flux sensor array", Measurement Science and Technology, (2010);21: 105201.

[5] D. Hubble and T. Diller, "Development and Evaluation of the Time-Resolved Heat and Temperature Array", Journal of Thermal Science and Engineering Applications, (2010).

[6] Y. Fu-en, "The heat flux measurement method and circular foil heat flux transducer, Journal of Astronautic Metrology and Measurement", (1984).

[7] D M Smith , H Moody and C Wanstall, "The Design and Use of Calorimeters For Characterization of High-Enthalpy Flows in Arc-Heated Test Facilities", AIAA Paper, 5236, (2002).

[8] Y. Qing-tao, B. Han-chen, Z. Tao, Y. Juan and W. Hui, "Design \& Response Characteristics Analy sis of a Fast-response Sensor for Temp and Heat Flux Measurement", Acta Armamentarii, (2014), pp. 927-34.

[9] Economou EN, A HYBRID METHOD FOR MEASURING HEAT FLUX, Springer, (2010)

[10] L. Chu-ping, Editor, "Heat flux measurement in aerothermodynamics and thermal protection test", National Defense Industry Press, (2013).

\section{Authors}

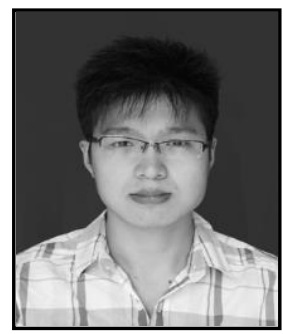

GUO Yu-yan, $\mathrm{He}$ received the B.E degrees from Henan Polytechnic University in 2012. From 2013 he is a student of master's course in School of Mechanical Engineering, Nanjing University of Science and Technology

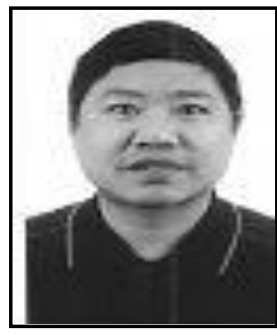

KONG De-ren, He received his B.S degree and $\mathrm{PhD}$ degree in measurement and control technology from Nanjing University of Science and Technology. He is a professor in Nanjing University of Science and Technology. His research interests include sensors and measurement.

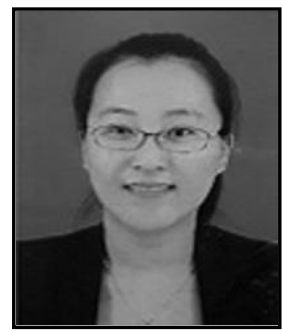

CHEN Jing, She received the B.E degrees from Nanjing University of Science and Technology in 2014. From 2014 he is a student of master's course in School of Mechanical Engineering, Nanjing University of Science and Technology 